\title{
Establishment of a novel system for the preoperative prediction of adherent perinephric fat (APF) occurrence based on a multi-mode and multi-parameter analysis of dual-energy CT
}

\author{
Guan $\mathrm{Li}^{1}$, Jie Dong ${ }^{2}$, Wei Huang ${ }^{1}$, Zhengyu Zhang ${ }^{2}$, Di Wang ${ }^{3}$, Mingyu Zou ${ }^{4}$, Qinmei Xu ${ }^{1}$, \\ Guangming Lu ${ }^{1}$, Zhiqiang $\mathrm{Cao}^{5}$ \\ ${ }^{1}$ Department of Radiology, ${ }^{2}$ Department of Urology, Jinling Hospital, Nanjing Medical University, Nanjing 210002, China; ${ }^{3}$ Department of \\ Pathology, ${ }^{4}$ Department of Radiology, ${ }^{5}$ Department of Urology, General Hospital of Northern Theater Command, Shenyang 110016 , China \\ Contributions: (I) Conception and design: All authors; (II) Administrative support: Z Cao, G Lu; (III) Provision of study material or patients: G Li, \\ J Dong; (IV) Collection and assembly of data: W Huang, Z Zhang; (V) Data analysis and interpretation: D Wang, M Zou; (VI) Manuscript writing: \\ All authors; (VII) Final approval of manuscript: All authors. \\ Correspondence to: Zhiqiang Cao. Department of Urology, General Hospital of Northern Theater Command, Shenyang 110016, China. \\ Email: 13309888392@163.com; Guangming Lu. Department of Radiology, Jinling Hospital, Nanjing Medical University, Nanjing 210002, China. \\ Email: cjr.luguangming@vip.163.com.
}

Background: Adherent perinephric fat (APF) is evaluated preoperatively with the Mayo adhesive probability (MAP) scoring system using conventional single-form computed tomography (CT) images. An objective or quantitative indicator for predicting APF is urgently needed for clinical application.

Methods: A total of 150 patients with renal tumours who underwent laparoscopic partial nephrectomy (LPN) were retrospectively enrolled and divided into the APF group $(n=100)$ and the non-APF group $(n=50)$ according to surgical results. All patients underwent a renal contrast-enhanced dual-energy CT (DECT) scan. The obtained CT DICOM data were transmitted to the DECT post-processing workstation and adopted virtual non-contrast (VNC), Rho/Z Maps, and Monoenergetic Plus (mono+) modes separately to undergo a multi-parameter analysis. A logistic stepwise investigation was utilized to analyse the related risk factors. The cutoff value was determined by the Youden index. Fifty patients were prospectively enrolled to validate the constructed model. The area under the curve (AUC), sensitivity, specificity and accuracy of the model were calculated.

Results: The study demonstrated that age, sex, body mass index (BMI), smoking status, tumour diameter, exophytic status, degree of malignancy and posterior perinephric fat thickness were related to the occurrence of APF $(\mathrm{P}<0.05)$. Model 1 was selected with the contrast material $(\mathrm{CM})$ parameter (cutoff point 0.5$)$, model 2 was selected with the effective atomic number $\left(Z_{\text {eff }}\right)$ parameter (cutoff point 6.5), and model 3 was selected with the slope $\mathrm{K}(\mathrm{K})$ parameter (cutoff point -0.95). The AUC, sensitivity, specificity and accuracy of model 1 were $0.94,0.94,0.93$ and 0.94 , respectively; for model 2, they were $0.94,0.93,0.93$ and 0.96 , respectively; and for model 3, they were $0.92,0.92,0.93$ and 0.92 , respectively.

Conclusions: Multi-mode and multi-parameter models of DECT can effectively be used to predict the occurrence of APF.

Keywords: Dual-energy CT (DECT); multi-mode; multi-parameter; adherent perinephric fat (APF); nephronsparing surgery (NSS)

Submitted Jun 28, 2019. Accepted for publication Sep 10, 2019.

doi: $10.21037 /$ tau.2019.09.23

View this article at: http://dx.doi.org/10.21037/tau.2019.09.23 


\section{Introduction}

In recent years, nephron-sparing surgery (NSS) has become the preferred method for stage T1N0M0 renal cell carcinoma (RCC) (1). An accurate preoperative assessment is one of the successful conditions for performing NSS (2). In 2009, Kutikov et al. first proposed a standardized renal tumour scoring system, the RENAL nephrometry score, which was used to quantify preoperative renal tumour characteristics (3). Subsequently, several methods, such as the PADUA, DAP and C-INDEX nephrometry scoring systems, were developed to evaluate the characteristics of preoperative renal tumours (4-6). However, these scoring systems have not considered the impact of the surrounding kidney conditions. When adherent perinephric fat (APF) occurs, it might increase the difficulty of partial nephrectomy (PN), even forcing clinicians to switch from $\mathrm{PN}$ to radical nephrectomy (RN). Previous studies have described that APF, characterized as inflammatory adipose tissue sticking to the kidneys, is challenging to surgeons performing NSS because it can increase the difficulty of tumour identification and exposure and is associated with a long duration of surgery and blood loss $(7,8)$.

Until 2014, Davidiuk et al. first proposed the effect of APF on PN and established a CT imaging-based Mayo adhesive probability (MAP) score to predict the occurrence of APF (9). However, the MAP score was evaluated on traditional CT images in which the form was single and the information provided was limited. Furthermore, the MAP score has a highly subjective effect in evaluating the perinephric fat stranding grades. The judgement of mild or severe perinephric stranding varies according to the degree of knowledge of different doctors. This one point will affect the accuracy of the preoperative evaluation. Therefore, an objective or quantitative indicator for predicting APF is urgently needed for clinical application.

\section{Methods}

\section{Study design and population}

Patients ( $\mathrm{n}=150 ; 94$ males and 56 females, with median age of $58.5 \pm 11.64$ years; range $32-85$ years) with renal tumours who underwent laparoscopic partial nephrectomy (LPN) in our institution were reviewed retrospectively between November 2017 and December 2018. As per the surgical records, 150 patients were divided into two groups based on whether APF occurred, namely, the APF group $(n=100)$ and the non-APF group $(n=50)$. Prospective patients $(\mathrm{n}=50 ; 34$ males and 16 females, with a median age of $52.5 \pm 12.47$ years; range $33-72$ years) with renal tumours who underwent LPN in our institution between January 2019 and April 2019 were reviewed. Two senior surgeons performed all surgeries. Surgeon's experience was categorized according to the number of LPNs performed: junior (during the first 50 cases), middle (between 50 and 100 cases), and senior (after the surgeon had completed 100 cases and above). Our institutional review board endorsed the prior study as a retrospective study, and informed consent was waived. After the study was prospectively validated, it was approved by the Ethics Committee of our hospital, and all patients gave informed consent to participate in the study before the examination.

\section{Inclusion and exclusion criteria}

The inclusion criteria were as follows: (I) initial diagnosis of a stage T1 renal tumour by ultrasonography, CT or magnetic resonance imaging (MRI); (II) underwent a renalenhanced dual-energy CT (DECT) examination before the operation; (III) LPN was performed by the same surgeon; and (IV) definitive surgical records for the diagnosis of APF or non-APF.

The exclusion criteria were as follows: (I) contrast agent allergy and other contraindications; (II) secondary surgery due to renal tumour recurrence; and (III) the perinephric space was completely occupied by tumours or the perinephric space was less than $0.5 \mathrm{~cm}^{2}$.

\section{DECT technique and reconstruction}

A renal three-phase dynamic contrast-enhanced scan was performed by DECT (SOMATOM Definition Flash, Siemens Healthcare, Forchheim, Germany). The scanning parameters were as follows: tube voltage and tube current (tube A $100 \mathrm{kVp}, 149$ eff.mAs and tube B Sn $140 \mathrm{kVp}$, 117 eff.mAs); gantry rotation time, $0.5 \mathrm{~s}$; detector configuration, $64 \times 0.6 \mathrm{~mm}$; and pitch, 1.2 . Automatic current modulation (CareDose4D; Siemens Healthcare) was used for all scans. The contrast agent iohexol (Omnipaque 350, GE Healthcare, USA) was injected into the anterior cubital vein with a dual-cylinder high-pressure syringe (Urich Medical, USA) at a rate of $4 \mathrm{~mL} / \mathrm{s}$ and a dose of $1.5 \mathrm{~mL} / \mathrm{kg}$, with a total volume of approximately $60-80 \mathrm{~mL}$. The region of interest (ROI) was placed at the branch level of the abdominal aorta and renal artery by using bolus tracking software to trigger. When the threshold reached $100 \mathrm{HU}$, a 
renal arterial phase CT scan was performed after a delay of $7 \mathrm{~s}$, followed by a delay of $40 \mathrm{~s}$ and $5 \mathrm{~min}$ for renal venous phase and delayed phase scanning, respectively. All images adopted a thickness of $0.75 \mathrm{~mm}$ and an interval of $0.5 \mathrm{~mm}$ with a kernel of Q30f to finish the reconstruction.

\section{DECT's multi-mode and multi-parameter analysis}

All renal venous phase CT data were transmitted to the DECT post-processing software package (syngo.via, version VB10B, Siemens Healthcare). The ROI measurement was performed using three modes: virtual non-contrast (VNC), Rho/Z Maps, and Monoenergetic Plus (mono+). In VNC mode, three parameters [e.g., VNC, contrast material $(\mathrm{CM})$ and mixed] were selected to measure the ROI. The VNC value represents the CT value of the virtual plain scan, the mixed value corresponds to the $\mathrm{CT}$ value of single energy after the fusion of two voltages, and the $\mathrm{CM}$ value represents the CT value of the target substance after the enhanced CT scan, and it reflects changes in CT values after material enhancement. Rho/Z Maps mode uses two parameters (e.g., Rho and Zeff) to measure the ROI. The Rho value is the number of electrons per unit volume, which is the electron density of normal water. Zeff represents the number of protons in the nucleus of a substance and can be used as a chemical quantification analysis. In Monoenergetic Plus mode, the slope of the curve [slope $(\mathrm{K})$ ] was calculated according to the formula $\mathrm{K}=1 \mathrm{HU} 40 \mathrm{keV}-\mathrm{HU} 110 \mathrm{keV}$ । / $110 \mathrm{keV}-40 \mathrm{keV}$. The slope (K) can be used to identify different chemical compositions of matter. Measurements were performed in consensus among two doctors (one was a radiologist, and the other was a urologist, with 10 and 20 years of work experience, respectively) to assure the appropriate placement of the ROIs. The mean \pm standard deviation (SD) was recorded.

The selection criteria of the ROIs were as follows (ROI1-3 were the APF group; ROI4-6 were the non-APF group): (I) ROI1: selected perinephric fat around the renal tumour appears to be the most prominent stranding and inflamed area (note: avoiding other neighbouring tissue, tumour tissue and tumour regenerative blood vessels); (II) ROI2: selected the other side of the perinephric fat; priority was selected if there were stranding and inflamed areas; (III) ROI3: selected the subcutaneous fat area of the lateral abdominal wall of the renal tumour; (IV) ROI4: selected in the same way as ROI1; perinephric fat around the renal tumour was selected if there were no stranding and inflamed areas; (V) ROI5: selected in the same way as ROI2; and
(VI) ROI6: selected in the same way as ROI3. The sizes (area $=0.5 \mathrm{~cm}^{2}$ ), shapes and locations of the ROIs remained consistent (Figure 1).

\section{Pathological analysis}

The pathological analysis of tumour and perirenal adipose tissue was performed on the 150 patients who underwent surgical resection. The specimens were fixed with formalin, dehydrated, embedded with paraffin, sectioned at a thickness of $4 \mathrm{~mm}$, and finally stained with conventional haematoxylin and eosin (H\&E) stain. A fluorescence biomicroscope (Olympus BX43) was used to observe the slices. The field of view was selected as " $\times 40, \times 100$ ".

\section{Statistical analysis}

\section{Retrospective study}

Statistical analyses were performed with IBM SPSS Statistics (version 22.0.0, Armonk, New York). Regarding measurement data: (I) those with a normal distribution are expressed as the mean $\pm \mathrm{SD}$; and (II) those with a non-normal distribution are expressed as the median (interquartile range) [M (IQR)]. Count data are expressed as the number of cases and the percentage $[\mathrm{n}(\%)]$. Univariate logistic regression models were used to analyse each factor. Odds ratios (ORs) and $95 \%$ confidence intervals (CIs) are given. Statistically significant variables $(\mathrm{P}<0.05)$ were included in the multivariate logistic regression model. Logistic regression with the forward stepwise method was used to analyse the significant variables $(\mathrm{P}<0.001)$ by the likelihood ratio test. Various parameters in multimode were selected as variables. The model containing the above independent predictors is presented in the form of a receiver operating characteristic (ROC) curve, and the best area under the curve (AUC) is used to demonstrate the diagnostic performance of the model. The maximum Youden index was used as the best cutoff point. The sensitivity, specificity, and accuracy were calculated to assess the efficiency of the model. A $\mathrm{P}$ value $<0.05$ was considered statistically significant.

\section{Prospective study}

The consistency evaluation of the measurement data from two doctors was analysed by the intraclass correlation coefficient (ICC) and calculated as the mean value. The AUCs of the MAP score and models were calculated and compared with Delong's test. The sensitivity, specificity, 

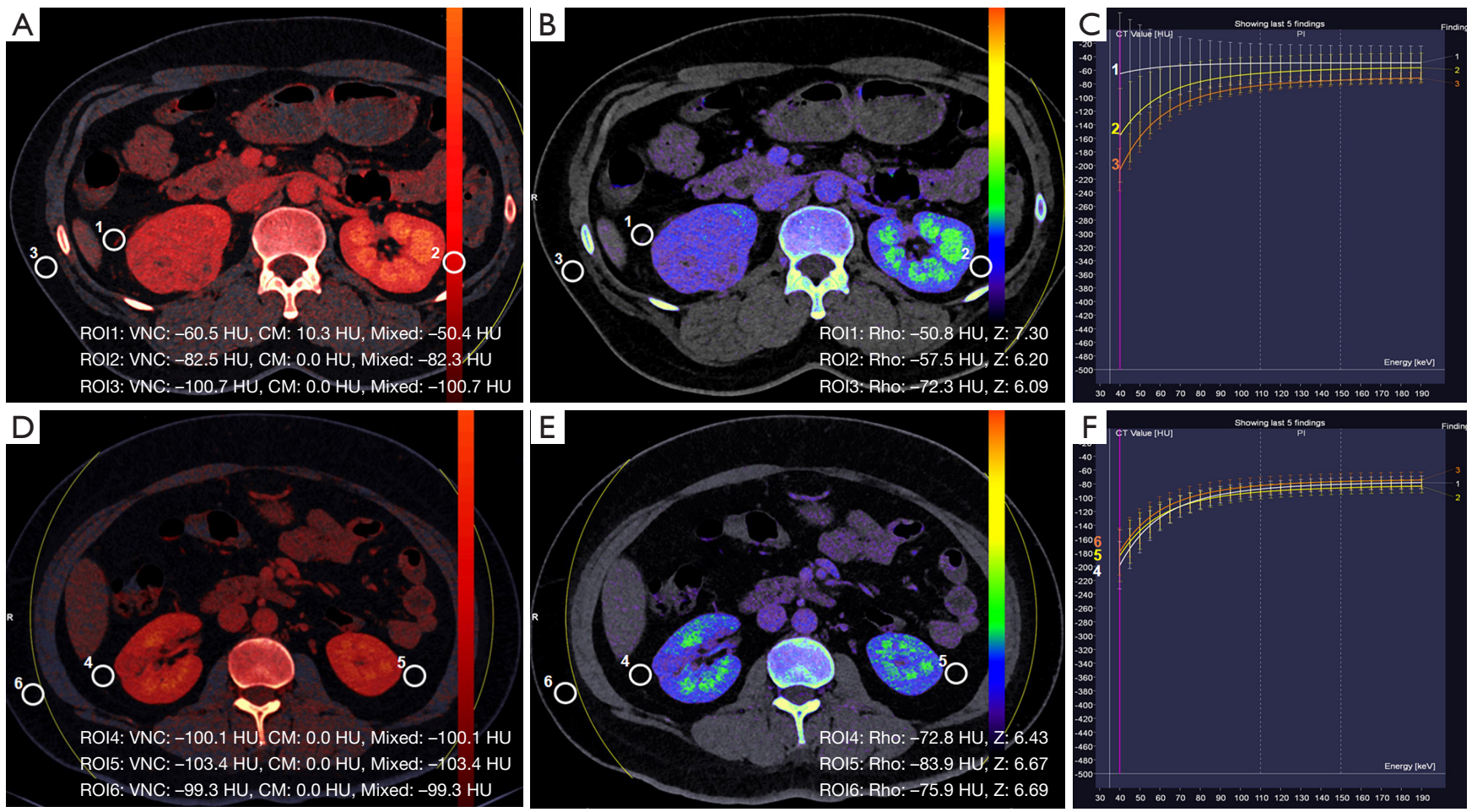

Figure 1 Multi-mode and multi-parameter analysis of dual-energy CT (DECT) in the adherent perinephric fat (APF) and non-APF groups. (A), (B) and (C) are the APF group; (D), (E) and (F) are the non-APF group. (A) and (D): virtual non-contrast (VNC) mode was used to measure the VNC, contrast material (CM) and mixed values of the ROIs; (B) and (E): Rho/Z mode was used to measure the Rho and Zeff values of the ROIs; (C) and (F): Monoenergetic Plus (mono+) mode was used to measure the slope (K) of the ROIs.

and accuracy of the MAP score and models were analysed with McNemar's test. The AUC, sensitivity, specificity, and accuracy of the MAP score and model intergroup comparisons were determined with Bonferroni correction, A $\mathrm{P}$ value $<0.05 / 3=0.0167$ was considered statistically significant.

\section{Results}

Table 1 summarizes the demographic characteristics of the cohort. Patients with APF were significantly older $(55.42 \pm 10.95$ vs. $46.12 \pm 11.44$ years, for non-APF patients, $\mathrm{P}<0.001)$ and had a higher BMI $(30.64 \pm 4.14$ vs. 26.02 \pm 4.66 , for non-APF patients, $\mathrm{P}<0.001)$. Furthermore, the proportion of males and smokers was higher in the APF group than in the non-APF group $(73 \% v s .32 \%, 66 \% v s$. $34 \%, \mathrm{P}<0.001)$.

Table 2 shows an evaluation of the correlation between tumour characteristics and APF. The largest range of renal tumour diameters was $4-7 \mathrm{~cm}(48 \%)$ in the APF group and smaller than $4 \mathrm{~cm}(64 \%)$ in the non-APF group. Renal tumours with more than $50 \%$ exogenous tumours were malignant, and those in the APF group were significantly higher than those in the non-APF group (63\% vs. 26\%, $\mathrm{P}<0.001)$. The proportion of malignant tumours in the APF group was also higher than that in the non-APF group (94\% vs. $58 \%, \mathrm{P}<0.001)$.

Table 3 reveals the relevant fat thickness between the $\mathrm{APF}$ and non-APF groups. The posterior perinephric fat thickness of the APF group was greater than that of the non-APF group $(1.69 \pm 0.74$ vs. $0.91 \pm 0.58, \mathrm{P}<0.001)$.

\section{Logistic regression analysis and model establishment}

The single-variable logistic regression analysis of multiple parameters in the DECT multi-mode showed that the VNC, CM, and mixed modes and Rho, $\mathrm{Z}$ and $\mathrm{K}$ parameters were all able to effectively distinguish between the APF group (ROI1) and the non-APF group (ROI4) $(\mathrm{P}<0.001)$ (Table 4 and Figure 1). Logistic regression was adopted with 
Table 1 Comparison of demographic characteristics between the adherent perinephric fat (APF) and non-APF groups

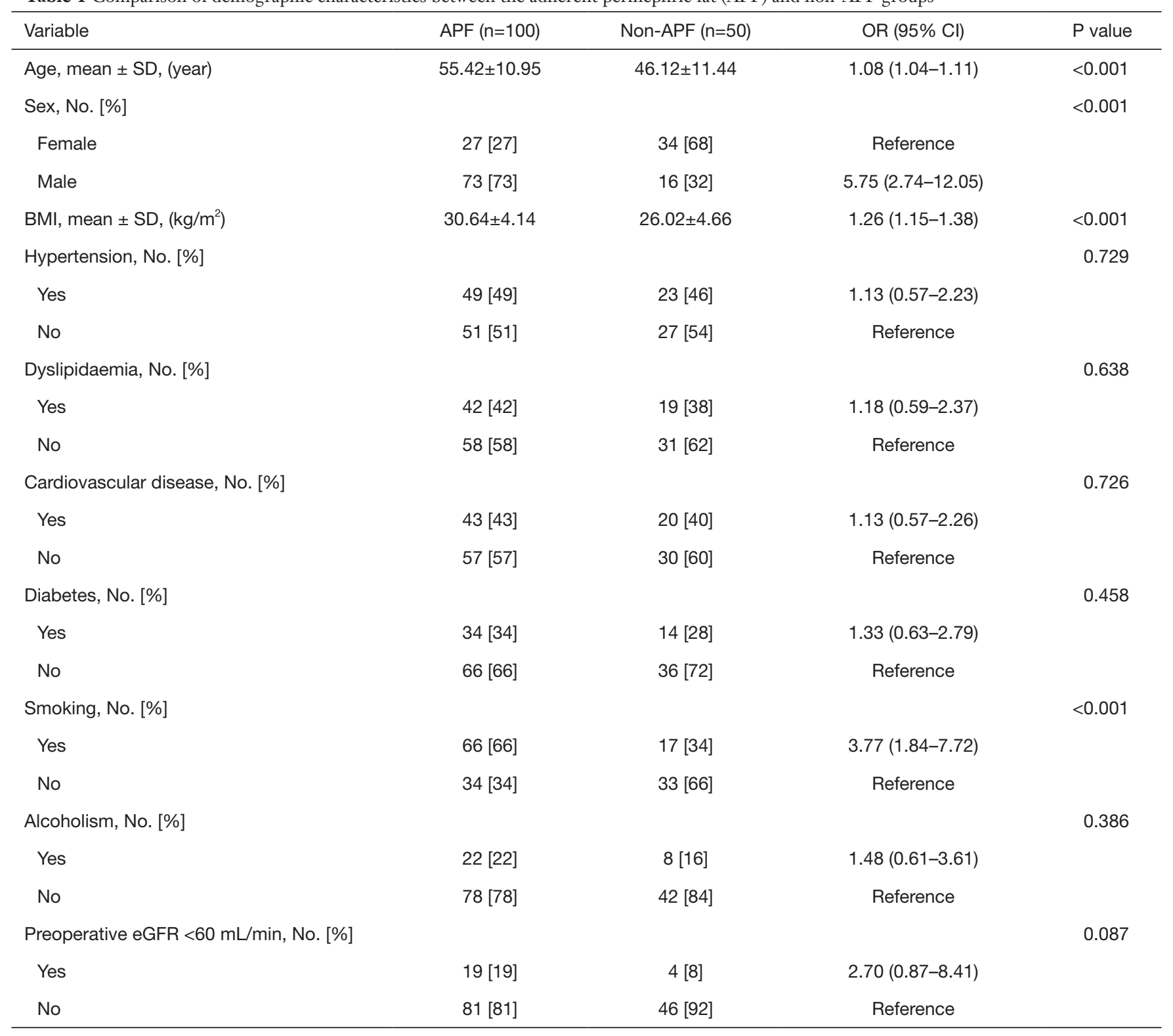

forward stepwise and selected more clinically meaningful parameters to build the final model: model 1 selected the CM (cutoff point 0.5 ), model 2 selected Zeff (cutoff point 6.5 ), and model 3 selected $\mathrm{K}$ (cutoff point -0.95 ). The model categories and $95 \%$ CIs are given in Table 5. An ROC curve analysis was carried out on the established model. The AUC, sensitivity, specificity and accuracy of model 1 were 0.99 (95\% CI, 0.98-1.00), 0.94 (95\% CI, 0.84-0.98), 0.98 (95\% CI, 0.95-1.00), and 0.95 (95\% CI, 0.93-0.99), respectively; those of model 2 were 0.98 (95\% CI,
0.97-1.00), 0.93 (95\% CI, 0.81-0.97), 0.97 (95\% CI, 0.920.99 ), and 0.95 (95\% CI, 0.91-0.98), respectively; and those of model 3 were 0.97 (95\% CI, 0.95-0.99), 0.98 (95\% CI, 0.90-1.00), 0.95 (95\% CI, 0.89-0.98), and 0.96 (95\% CI, 0.92-0.98), respectively (Figure 2).

\section{Prospective validation of the three models}

The ICC (95\% CI) result of the consistency evaluation of two doctors was $0.86(0.40-0.97)$. All patients were measured 
Table 2 Comparison of tumour characteristics between the adherent perinephric fat (APF) and non-APF groups

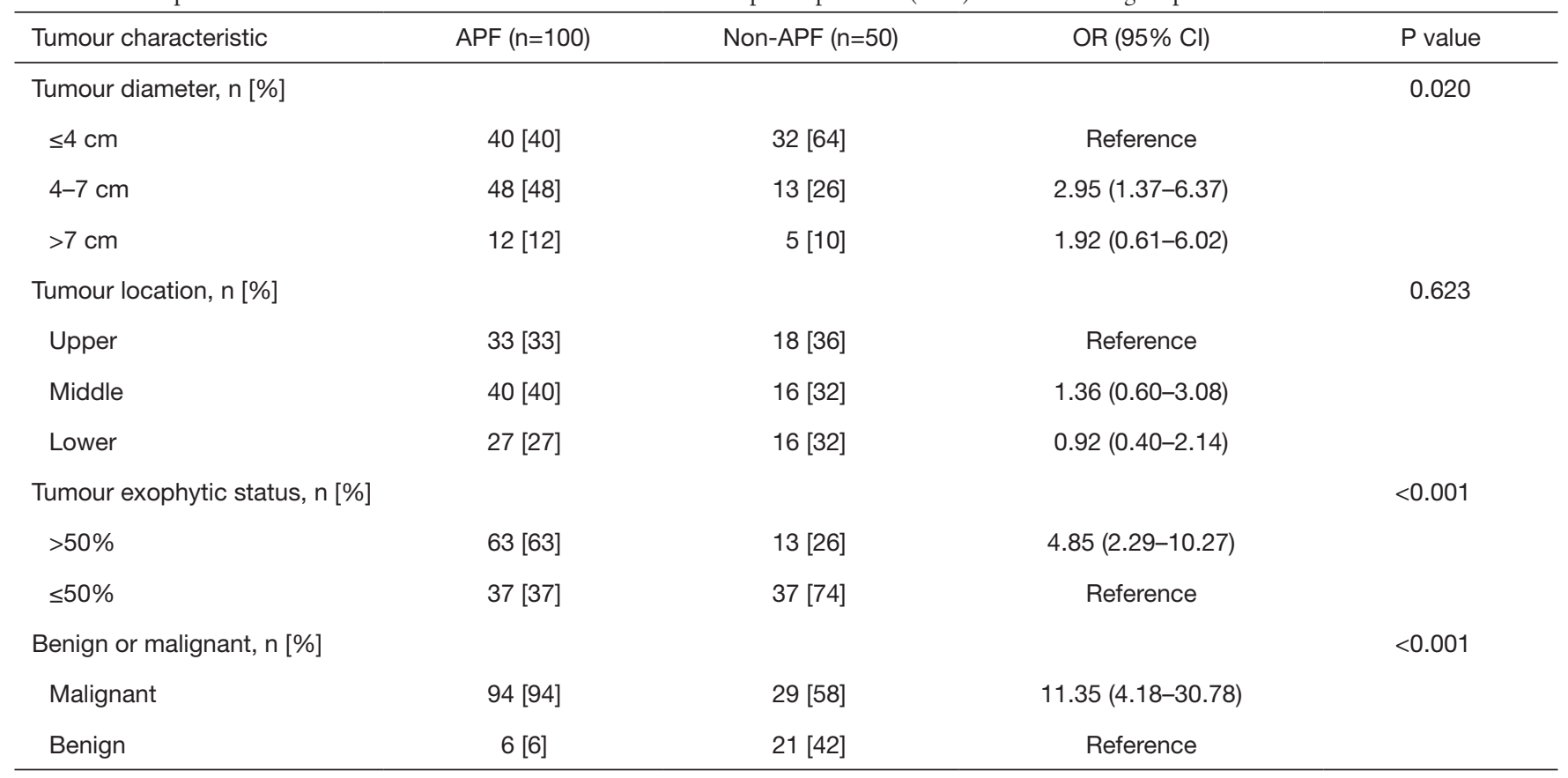

Table 3 Comparison of relevant perinephric fat thicknesses between the adherent perinephric fat (APF) and non-APF groups

\begin{tabular}{|c|c|c|c|c|}
\hline Relevant fat thickness & APF $(n=100)$ & Non-APF $(n=50)$ & OR $(95 \% \mathrm{Cl})$ & $P$ value \\
\hline Posterior perinephric fat thickness (cm) & $1.69 \pm 0.74$ & $0.91 \pm 0.58$ & $5.55(2.91-10.59)$ & $<0.001$ \\
\hline Subcutaneous fat thickness of the lateral abdominal wall $(\mathrm{cm})$ & $1.45 \pm 0.39$ & $1.47 \pm 0.29$ & $0.872(0.34-2.22)$ & 0.774 \\
\hline
\end{tabular}

Table 4 DECT multi-parameter single-variable logistic regression analysis between the adherent perinephric fat (APF) and non-APF groups

\begin{tabular}{|c|c|c|c|c|}
\hline Parameter & APF (ROI1) & Non-APF (ROI4) & OR $(95 \% \mathrm{Cl})$ & $P$ value \\
\hline $\mathrm{CM}$ & $8.50(5.03-10.30)$ & $0.55(0.00-2.30)$ & $1.412(1.216-1.640)$ & $<0.001$ \\
\hline Mixed & $-55.45(-64.40$ to -47.83$)$ & $-96.30(-99.98$ to -69.60$)$ & $1.212(1.131-1.298)$ & $<0.001$ \\
\hline Rho & $-49.40(-54.55$ to -41.25$)$ & $-72.25(-78.35$ to -57.83$)$ & $1.226(1.139-1.321)$ & $<0.001$ \\
\hline K & $-0.23(-0.37$ to -0.10$)$ & $-1.10(-1.30$ to -0.72$)$ & $1.594(1.380-1.841)$ & $<0.001$ \\
\hline
\end{tabular}

ORs and $95 \% \mathrm{Cls}$ were estimated from single-variable logistic regression models. 
Table 5 Model establishment, categories and 95\% CIs for the prediction of APF occurrence

\begin{tabular}{lcccr}
\hline Pattern & Variable & Category & Fraction (\%) & \multicolumn{1}{c}{$95 \% \mathrm{Cl}$} \\
\hline Model 1 & $\mathrm{CM}$ & $<0.5$ & $1 / 48(2.1)$ & $0.4-10.9$ \\
& & $\geq 0.5$ & $99 / 102(97.1)$ & $91.7-99.0$ \\
Model 2 & \multirow{2}{*}{$\mathrm{Z}_{\text {eff }}$} & $<6.5$ & $2 / 48(4.17)$ & $1.2-14.0$ \\
& & $\geq 6.5$ & $98 / 102(96.1)$ & $90.3-09.5$ \\
Model 3 & $\mathrm{K}$ & $<-0.95$ & $5 / 54(9.3)$ & $4.0-19.9$ \\
& & $\geq-0.95$ & $95 / 96(99.0)$ & $94.3-99.8$ \\
\hline
\end{tabular}

APF, adherent perinephric fat; CM, contrast material.

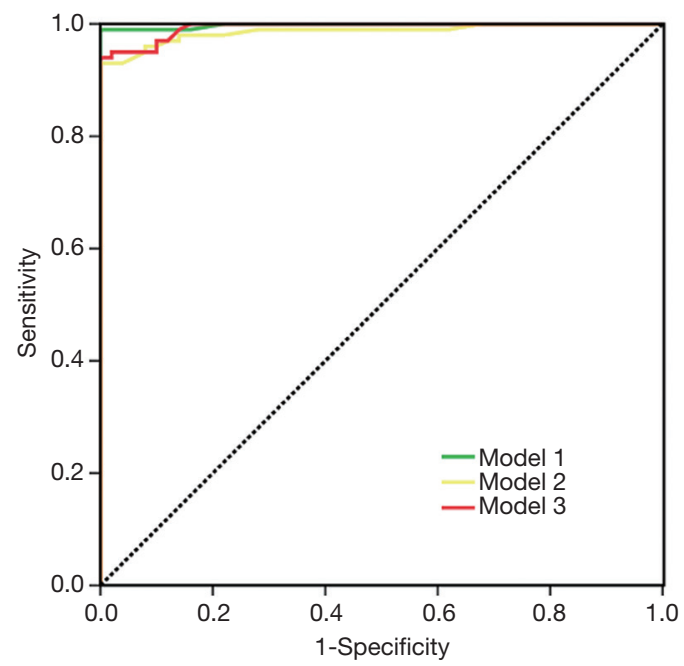

Figure 2 Receiver operating characteristic (ROC) curve analysis of the three models.

by the MAP score. The three models had a higher AUC, specificity, sensitivity, and accuracy than the MAP score. The $P$ values of the AUC, sensitivity, specificity, and accuracy of the MAP score and models were all $<0.0167$ (Table 6).

\section{Comparison of multiple parameters of different ROIs}

The ROI measurement results showed significant differences in the $\mathrm{CM}, \mathrm{Z}$ and $\mathrm{K}$ values of ROI1, ROI2 and ROI3 in the APF group (all $\mathrm{P}<0.001$ ). In the non-APF group, the $\mathrm{CM}, \mathrm{Z}$ and $\mathrm{K}$ values of ROI4 and ROI5 had no significant difference (all $\mathrm{P}>0.999$ ), while ROI4 or ROI5 differed from ROI6 $(\mathrm{P}>0.05)$. The $\mathrm{CM}, \mathrm{Z}$ and $\mathrm{K}$ values of ROI1 were significantly different from those of ROI4 (all $\mathrm{P}<0.001)$, and ROI2 differed from ROI5 $(\mathrm{P}<0.001,0.013$ and $<0.001)$, but there was no difference between ROI3 and ROI6 ( $\mathrm{P}=0.634,0.174$ and 0.828$)$.

\section{Pathological analysis results}

Figure 3 displays the gross samples of the APF and non-APF groups. While no obvious differences were discernible by the naked eye, microscopic changes in neovascularization, hyperaemia with a small amount of lymphocyte infiltration, and different degrees of fibrous tissues in the APF group were observed. Normal adipocytes were found only in the non-APF group.

\section{Objective evaluation}

Table 7 summarizes the perioperative outcomes in the cohort. DECT's multi-model analysis of patients with APF predicted a significantly shorter operative time (62.3 vs. $97.2 \mathrm{~min}, \mathrm{P}<0.001)$ and less estimated blood loss (18.5 vs. $45.9 \mathrm{~mL}, \mathrm{P}<0.05)$ than those without APF. No significant difference was found in ischaemia time $(\mathrm{P}=0.063)$, hospital stay $(\mathrm{P}=0.218)$, or postoperative complications $(\mathrm{P}=0.158)$.

\section{Discussion}

The preoperative accurate prediction of APF can be helpful for the formulation of surgical planning, the assessment of surgical risks, and the reduction in surgical complications. The current study showed that age, sex, BMI, smoking status, tumour diameter, exophytic status, benign or malignant degree and posterior perinephric fat thickness were significantly related to the occurrence of APF (all $\mathrm{P}<0.05$ ). Although these indicators were not included in the final model, they can be used as risk factors to predict APF. We demonstrated that DECT's multi-mode and multiparameter models can be effectively applied to predict APF. The prospective validation revealed that the three models had a higher AUC, specificity, sensitivity, and accuracy than the MAP score $(\mathrm{P}<0.01)$.

The present study also showed that APF has a certain correlation with $\mathrm{BMI}$; the higher the $\mathrm{BMI}$ is, the more it is susceptible to APF. Lee et al. reported that APF was related to metabolic syndrome (MetS), which includes a range of disorders such as glucose intolerance, dyslipidaemia, and hypertension (10). A high BMI and being overweight can lead to MetS $(11,12)$. Our study showed that patients who smoke have a high risk of APF, which is consistent with the findings of Davidiuk et al. (9). 
Table 6 Prospective validation diagnostic efficacy of the three models and the MAP score

\begin{tabular}{lcccc}
\hline Pattern & AUC $(95 \% \mathrm{Cl})$ & Sensitivity $(95 \% \mathrm{Cl})$ & Specificity $(95 \% \mathrm{Cl})$ & Accuracy $(95 \% \mathrm{Cl})$ \\
\hline Model 1 & $0.94(0.85-1.00)$ & $0.94(0.82-0.98)$ & $0.93(0.69-0.99)$ & $0.94(0.84-0.98)$ \\
Model 2 & $0.94(0.85-1.00)$ & $0.93(0.80-0.98)$ & $0.93(0.69-0.99)$ & $0.96(0.87-0.99)$ \\
Model 3 & $0.92(0.83-1.00)$ & $0.92(0.78-0.97)$ & $0.93(0.69-0.99)$ & $0.92(0.81-0.97)$ \\
MAP score & $0.85(0.78-092)$ & $0.84(0.85-0.96)$ & $0.86(0.74-0.93)$ & $0.85(0.78-0.90)$ \\
\hline
\end{tabular}
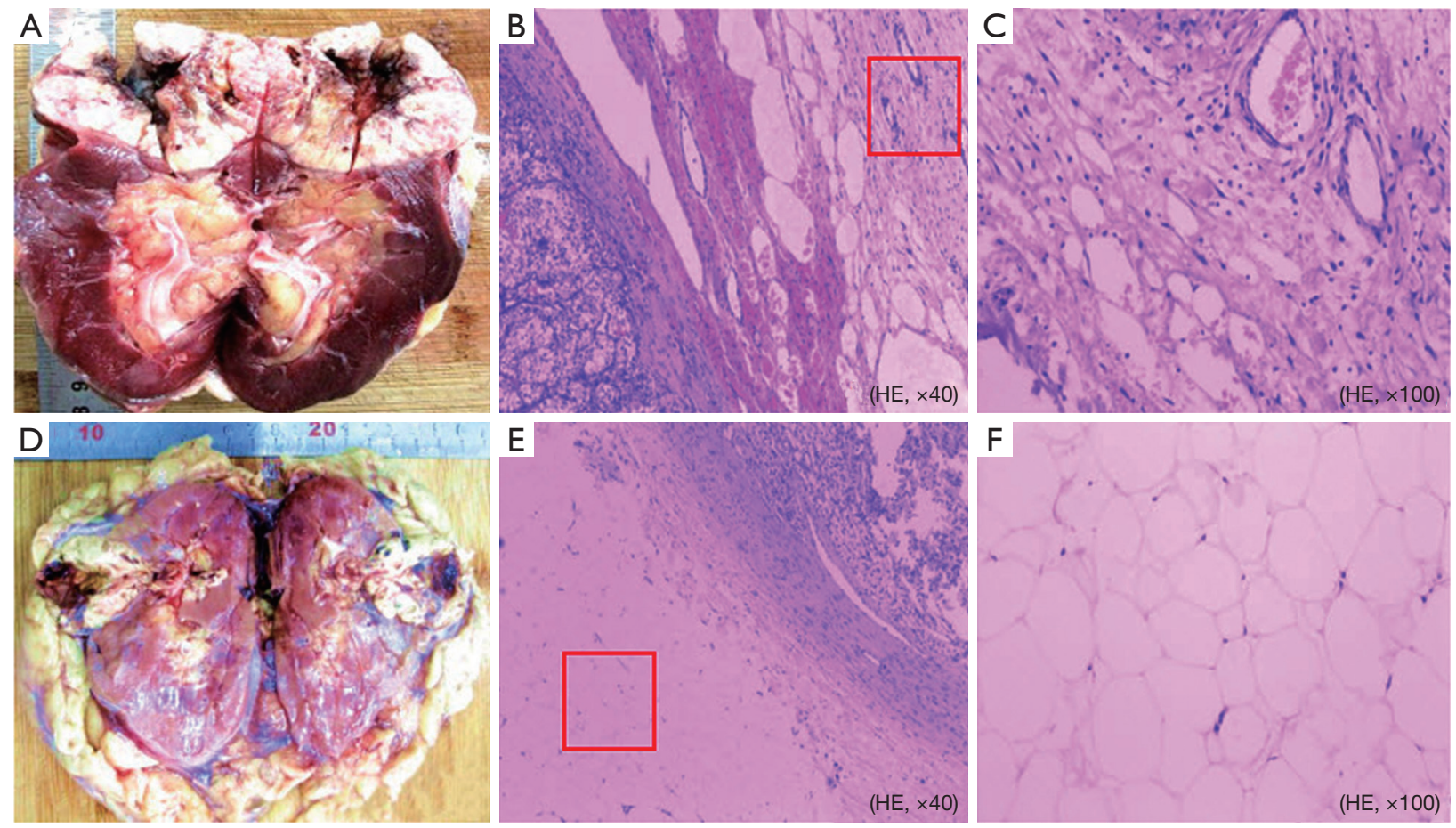

Figure 3 Gross and microscopic images of APF and non-APF. Panels (A) and (D) show the gross appearance of APF and non-APF. Panels (B) and $(\mathrm{C})$ and panels $(\mathrm{E})$ and $(\mathrm{F})$ are representative photomicrographs $(\mathrm{HE}, \times 40, \times 100)$ of $\mathrm{APF}$ and non-APF, respectively. Panels $(\mathrm{C})$ and $(\mathrm{F})$ show the areas corresponding to the red box in panels (B) and (E), respectively.

Furthermore, Cheng et al. proposed that cigarette smoking was associated with MetS and that smokers had a higher BMI than non-smokers (13).

The mechanism of APF is still unclear. Studies have reported that APF may be related to the inflammatory microenvironment, MetS, the autoimmune response and idiopathic fibrosis (14-16). Activation of the chronic inflammation system can cause endothelial cells to secrete a variety of cytokines, chemokines, and plasminogen activator inhibitor type 1 (PAI-1), thereby reducing fibrinolytic activity and leading to fibrous adhesion $(17,18)$. Serotonin [5-hydroxytryptamine (5-HT)], which is considered a fibrosis-related growth factor and contributor to APF, is highly expressed when RCC occurs $(19,20)$. When an exophytic tumour is $>50 \%$, the renal tumour invades the perinephric fat, so the release of serotonin from the RCC can act more directly on perinephric fat, leading to the occurrence and aggravation of APF. Furthermore, Duong et al. proposed that adipose tissue (AT) and its main cellular component, adipocytes, are key actors in solid tumour progression (21). Thus, APF can act on kidney tumours to increase their diameter. Our study found that the probability of APF in benign renal tumours is lower than that in malignant tumours. The reason for this finding may be that malignant tumours are more aggressive and metastatic than benign tumours. Although the exophytic 
Table 7 Comparison of perioperative outcomes between DECT's multi-model group and the control group

\begin{tabular}{|c|c|c|c|c|}
\hline Variable & DECT's multi-model group $(n=50)$ & Control group $(n=50)$ & $\mathrm{t}$ value & $P$ value \\
\hline Ischaemia time, min & $26.9 \pm 1.2$ & $27.3 \pm 0.9$ & 1.886 & 0.063 \\
\hline $\mathrm{EBL}, \mathrm{mL}$ & $18.5 \pm 9.4$ & $45.9 \pm 10.1$ & 14.042 & $<0.001$ \\
\hline Hospital stay, days & $6.0 \pm 1.4$ & $6.4 \pm 1.8$ & 1.240 & 0.218 \\
\hline
\end{tabular}

DECT, dual-energy CT; OR, operative; EBL, estimated blood loos.

tumour is larger, the chances of developing APF are also greater. However, when the tumours are too large and the perirenal fat is almost completely occupied by the tumours, it is difficult to select the ROI, which leads to false positives.

With the advent of DECT and the development of postprocessing software functions, more information is obtained compared to traditional single-energy CT (22). DECT is equipped with two $\mathrm{X}$-ray tube and detector systems, and the two tubes simultaneously emit $\mathrm{X}$-rays at the respective set tube voltages so two groups of dual-energy data can be obtained (23). DECT can be used to analyse the chemical composition, properties, and content of substances (24). The common post-processing software package includes VNC, Rho/Z Maps and Monoenergetic Plus (mono+) modes. VNC mode directly adopts a DECT contrastenhanced CT scan without performing a standard noncontrast (SNC) CT scan (25). VNC mode can obtain the change in the CT value before and after enhancement. Rho/Z Maps mode uses X-ray attenuation to calculate the atomic number of unknown elements. For a compound, if its attenuation effect is equivalent to an element, the atomic number can be considered the effective atomic number (Zeff) of the compound (26). Monoenergetic Plus (mono+) mode is based on the difference in the degree of X-ray absorption attenuation of different substances and generates a unique spectral curve (27). Different substances have their corresponding characteristic curves and slopes (28). The reason why ROIs are mainly measured in renal vein phaseenhanced images is because the kidney and perinephric tissues are clearly shown in this phase. The normal spectrum ranges from 40-190 keV. However, we chose the spectrum range from $40-110 \mathrm{keV}$ because within this range the curve shape and slope $(\mathrm{K})$ are highly characteristic.

The multiple parameter outcomes revealed that the CM, Zeff, and $\mathrm{K}$ of ROI1 in the APF group were significantly different from those of ROI4 in the non-APF group, which demonstrated that the three models could effectively distinguish APF from non-APF. The CM, Zeff, and K values of ROI1, ROI2, and ROI3 in the APF group were all significantly different, indicating that although they were all AT, they might have different compositions or contents. We found that perinephric fat was different from abdominal subcutaneous fat. When APF occurred, there was a simultaneous occurrence in both perinephric areas. In addition, renal tumours can aggravate the extent of APF.

There were a few limitations to our investigation. First, the sample size of this study was relatively small. The conclusions of this research need to be further studied in a larger data set. Second, the occurrence of APF can be confirmed by a surgeon's feedback and pathological analysis, but the gold standard method for determining the degree of APF is still lacking. Therefore, further research is needed to predict the degree of APF by the model. Third, the model results have not been compared to other kinds of DECTs from other manufacturers. For patients who have undergone a CT examination in an outpatient hospital, the popularization of DECT examination again has certain limitations. Finally, stepwise regression procedures have certain problems, such as instability of the selection and the biased estimation of coefficients.

\section{Conclusions}

DECT's multi-mode and multi-parameter models can effectively predict the occurrence of APF, and models combined with relevant clinical risk factors are expected to become a novel method for predicting the occurrence of APF.

\section{Acknowledgments}

Funding: This study was supported by the National Clinical 
Key Specialist Military Construction Project of China, the Clinical Medical Imaging Center Project of Jiangsu Province, China (grant YXZXA2016007) and the Integrated Research and Development of Hemostatic Drugs for War Trauma Treatment (grant 2016ZX09J16103).

\section{Footnote}

Conflicts of Interest: The authors have no conflicts of interest to declare.

Ethical Statement: The authors are accountable for all aspects of the work in ensuring that questions related to the accuracy or integrity of any part of the work are appropriately investigated and resolved. Approval for this research was obtained from the Ethics Committee of Jinling Hospital, Nanjing Medical University (2017NZGKJ-078), and patient consent was obtained.

\section{References}

1. Badalato GM, Kates M, Wisnivesky JP, et al. Survival after partial and radical nephrectomy for the treatment of stage T1bN0M0 renal cell carcinoma (RCC) in the USA: a propensity scoring approach. BJU Int 2012;109:1457-62 .

2. Petros FG, Keskin SK, Yu KJ, et al. Intraoperative Conversion From Partial to Radical Nephrectomy: Incidence, Predictive Factors, and Outcomes. Urology 2018;116:114-9.

3. Kutikov A, Uzzo RG. The R.E.N.A.L. nephrometry score: a comprehensive standardized system for quantitating renal tumor size, location and depth. J Urol 2009;182:844-53.

4. Ficarra V, Novara G, Secco S, et al. Preoperative aspects and dimensions used for an anatomical (PADUA) classification of renal tumours in patients who are candidates for nephron-sparing surgery. Eur Urol 2009;56:786-93.

5. Simmons MN, Hillyer SP, Lee BH, et al. Diameteraxial-polar nephrometry: integration and optimization of R.E.N.A.L. and centrality index scoring systems. J Urol 2012;188:384-90.

6. Simmons MN, Ching CB, Samplaski MK, et al. Kidney tumor location measurement using the $\mathrm{C}$ index method. J Urol 2010;183:1708-13.

7. Yao $Y$, Gong H, Pang Y, et al. Risk Factors Influencing the Thickness and Stranding of Perinephric Fat of Mayo Adhesive Probability Score in Minimally Invasive Nephrectomy. Med Sci Monit 2019;25:3825-31.
8. Khene ZE, Bensalah K, Largent A, et al. Role of quantitative computed tomography texture analysis in the prediction of adherent perinephric fat. World J Urol 2018;36:1635-42.

9. Davidiuk AJ, Parker AS, Thomas CS, et al. Mayo adhesive probability score: an accurate image-based scoring system to predict adherent perinephric fat in partial nephrectomy. Eur Urol 2014;66:1165-71.

10. Lee SM, Robertson I, Stonier T, et al. Contemporary outcomes and prediction of adherent perinephric fat at partial nephrectomy: a systematic review. Scand J Urol 2017;51:429-34.

11. Batsis JA, Mackenzie TA, Bartels SJ, et al. Diagnostic accuracy of body mass index to identify obesity in older adults: NHANES 1999-2004. Int J Obes (Lond) 2016;40:761-7.

12. Egert S, Baxheinrich A, Lee-Barkey YH, et al. Effects of a hypoenergetic diet rich in $\alpha$-linolenic acid on fatty acid composition of serum phospholipids in overweight and obese patients with metabolic syndrome. Nutrition 2018;49:74-80.

13. Cheng E, Burrows R, Correa P, et al. Light smoking is associated with metabolic syndrome risk factors in Chilean young adults. Acta Diabetol 2019;56:473-9.

14. Khene ZE, Peyronnet B, Mathieu R, et al. Analysis of the impact of adherent perirenal fat on peri-operative outcomes of robotic partial nephrectomy. World J Urol 2015;33:1801-6.

15. Macleod LC, Hsi RS, Gore JL, et al. Perinephric fat thickness is an independent predictor of operative complexity during robot-assisted partial nephrectomy. J Endourol 2014;28:587-91.

16. Timar O, Sestier F, Levy E. Metabolic syndrome X: a review. Can J Cardiol 2000;16:779-89.

17. Kosaka H, Yoshimoto T, Fujimoto J, et al. Interferongamma is a therapeutic target molecule for prevention of postoperative adhesion formation. Nat Med 2008;14:437-41.

18. Trayhurn P, Wood IS. Adipokines: inflammation and the pleiotropic role of white adipose tissue. Br J Nutr 2004;92:347-55.

19. Jungwirth N, Haeberle L, Schrott KM, et al. Serotonin used as prognostic marker of urological tumors. World J Urol 2008;26:499-504.

20. Chen L, Chen G, Guo Y, et al. Ketanserin, a serotonin $2 \mathrm{~A}$ receptor antagonist, alleviates ischemia-related biliary fibrosis following donation after cardiac death liver transplantation in rats. Liver Transpl 2014;20:1317-26. 
21. Duong MN, Geneste A, Fallone F, et al. The fat and the bad: Mature adipocytes, key actors in tumor progression and resistance. Oncotarget 2017;8:57622-41.

22. Goo HW, Goo JM. Dual-Energy CT: New Horizon in Medical Imaging. Korean J Radiol 2017;18:555-69.

23. Gabbai M, Leichter I, Mahgerefteh S, et al. Spectral material characterization with dual-energy CT: comparison of commercial and investigative technologies in phantoms. Acta Radiol 2015;56:960-9.

24. Wan Y, Guo H, Ji L, et al. Gemstone spectral imaging dual-energy computed tomography for differentiation of renal cell carcinoma and minimal-fat renal angiomyolipoma. J Cancer Res Ther 2018;14:S394-9.

25. Schwarz F, Nance JW Jr, Ruzsics B, et al. Quantification of coronary artery calcium on the basis of dual-energy

Cite this article as: $\mathrm{Li}$ G, Dong J, Huang W, Zhang Z, Wang D, Zou M, Xu Q, Lu G, Cao Z. Establishment of a novel system for the preoperative prediction of adherent perinephric fat (APF) occurrence based on a multi-mode and multiparameter analysis of dual-energy CT. Transl Androl Urol 2019;8(5):421-431. doi: 10.21037/tau.2019.09.23 coronary CT angiography. Radiology 2012;264:700-7.

26. Hua CH, Shapira N, Merchant TE, et al. Accuracy of electron density, effective atomic number, and iodine concentration determination with a dual-layer dualenergy computed tomography system. Med Phys 2018;45:2486-97.

27. Albrecht MH, Scholtz JE, Hüsers K, et al. Advanced image-based virtual monoenergetic dual-energy CT angiography of the abdomen: optimization of kiloelectron volt settings to improve image contrast. Eur Radiol 2016;26:1863-70.

28. Wang N, Ju Y, Wu J, et al. Differentiation of liver abscess from liver metastasis using dual-energy spectral CT quantitative parameters. Eur J Radiol 2019;113:204-8. 